\title{
Consumo de produtos e serviços inovadores: aplicação do índice de prontidão para tecnologia*
}

\author{
Luciana Peixoto Santa Rita \\ Universidade Federal de Alagoas (Ufal) \\ Alcides Carlos de Araújo \\ Universidade Federal de Alagoas (Ufal) \\ Mainah Almeida de Paula \\ Universidade Federal de Alagoas (Ufal) \\ Manuella de Oliveira Lima \\ Universidade de São Paulo (USP) \\ José Carlos Viana Filho \\ Universidade Federal de Alagoas (Ufal)
}

Recebido: 30/4/2008 Versão revisada (entregue): 16/04/2009 Aprovado: 22/04/2009

RESUMO

Este estudo teve como objetivo mensurar o índice de prontidão para tecnologia no contexto do Estado de Alagoas. O estudo baseou-se na escala Índice de Prontidão para Tecnologia (Technology Readiness Index - TRI) de Parasuraman (2000). Foi realizada uma pesquisa descritiva de natureza quantitativa, baseada em uma amostra probabilística, com procedimentos de coleta e análise estruturados. A amostra foi composta de 326 respondentes, com margem de erro de 5,4\% e nível de confiança de 95\%. Os resultados mostraram que lidar com tecnologia no trabalho e ter mais pessoas em casa usando tecnologia afeta a TRI do indivíduo. Como contribuição à academia, a pesquisa descreveu uma revisão e uma aplicação prática em relação ao fenômeno dos produtos de alta tecnologia e sua ligação com o processo de adoção.

* Gostaríamos de agradecer a todos os pareceristas, cientistas que despendem seu tempo precioso na leitura e avaliação de artigos especialmente designados pelo Conselho Editorial, pelos preciosos comentários e orientações. 
Luciana Peixoto Santa Rita, Alcides Carlos de Araújo, Mainah Almeida de Paula, Manuella de Oliveira Lima, José Carlos Viana Filho

PALAVRAS-ChAVE | comportamento do consumidor; inovação; TRI.

Códigos JEL | C12; C39; M31.

\title{
Consumption of innovative goods and services: application of the technology readiness index
}

\begin{abstract}
This study aimed at measuring the index of disposition towards technology in the context of the state of Alagoas in Brazil. The study was based on Parasuraman (2000) Technology Readiness Index (TRI). A descriptive research of quantitative nature was made, starting from a non probabilistic sample, with a structured collecting and analysis procedure. The sample was composed of 326 people, with an error margin of 5.4\% and a confidence level of $95 \%$. We noticed that people, who deal with technology at work, present a significantly higher TRI than those who don't. We have also observed that families using technology at home may significantly affect the TRI of its members. As a contribution to the academic world, the research described a revision and a practical application in relation to the phenomenon of high technology products and its connection to the process of adopting them.
\end{abstract}

KEYWORDS | consumer's behavior; innovation; TRI.

JEL CODES: C12; C39; M31.

\section{Introdução}

Cada vez mais, os consumidores se veem diante da necessidade de utilizar produtos mais complexos e a organização assume o papel de produzir bens e serviços com tecnologia suficiente para atender às expectativas deles. De acordo com Souza (2002), os avanços tecnológicos têm produzido considerável impacto sobre o modo como os consumidores lidam com produtos cada vez mais sofisticados e como os serviços são produzidos e entregues. Por essa perspectiva, considera-se de extrema importância investigar o perfil de consumo de alta tecnologia, a fim de detectar novas formas de comercialização com base nas demandas apresentadas pelo mercado. 
Segundo Cortada (2002), em geral, novos produtos encontram mercados dispostos a adquiri-los em quantidades crescentes e a preços mais elevados. Sendo assim, o investimento em tecnologia por parte da organização pode refletir em diferencial competitivo, tendo em vista as tendências à modernização dos padrões de consumo do mercado.

Entre as teorias que estudam o fenômeno, Parasuraman e Colby (2001) ressaltam que as ações inadequadas de marketing, em se tratando de produtos e serviços de alta tecnologia, são motivadas pela compreensão errônea das atitudes dos clientes em relação à tecnologia e às variaçóes dessas atitudes em cada segmento diferente de clientes.

Quanto a produtos naturalmente inovadores, que têm como característica evidente um ciclo de vida mais curto que os demais bens de consumo, são ainda desconhecidos muitos aspectos do comportamento do consumidor. O estudo de mercados de produtos de alta tecnologia desperta a atenção e o interesse não somente de pesquisadores como também e, sobretudo, de empresas e governos. Diante dessa afirmativa, compreende-se que a existência de mercados cada vez mais tecnológicos é um fato iminente, e conhecer o perfil dos demandantes desse mercado é fundamental para a capacidade inovadora das organizaçóes.

Este estudo teve como objetivo mensurar o índice de prontidão para tecnologia no contexto do Estado de Alagoas, por meio da reaplicação do instrumento de medida a uma amostra de 326 consumidores, a partir de 16 (dezesseis) anos, na cidade de Maceió. O desenvolvimento da pesquisa ocorreu com a investigação conduzida por Parasuraman (2000) e Parasuraman e Colby (2001), os quais identificaram que os consumidores, em seus comportamentos de compra de produtos e serviços baseados em tecnologia, podem ser identificados segundo um Índice de Prontidão para Tecnologia. Esse índice é denominado Technology Readiness Index (TRI) e caracterizou-se como um importante instrumento de medida da prontidão para tecnologia do consumidor norte-americano, tendo sido aplicado e validado naquele contexto. A principal motivação para a pesquisa deu-se mediante a constatação da necessidade de se conhecer o mercado consumidor high tech e proporcionar ao meio acadêmico, bem como às organizações envolvidas no processo de produção de bens e serviços inovadores, um conhecimento mais aprofundado dos hábitos de consumo de produtos e serviços de alta tecnologia. Espera-se, portanto, com a realização deste trabalho, oferecer contribuição relevante à ciência e ampliar os horizontes para novas investigaçōes na área estudada. Dividido em cinco seçoes, na introdução faz-se uma abordagem da problemática, sendo apresentados tema, justificativa e objetivos deste 
artigo. Em seguida, uma revisão da literatura apresenta a discussão do processo de inovação, adoção de compra e comportamento de consumo de produtos de alta tecnologia para mostrar a relevância e interseções desses temas. Posteriormente, há a descrição dos procedimentos metodológicos e a análise dos dados. A seguir, são apresentadas as considerações finais, incluindo suas implicações teóricas e práticas, bem como as limitaçōes e as recomendaçôes para novas pesquisas.

\section{Revisão conceitual}

$\mathrm{Na}$ literatura, pode-se observar o emprego de diversos referenciais para definir inovação e comportamento de consumo. Logo, a seção aborda diferentes contribuições do tema, abrangendo aspectos amplos, mas sem pretender descrever à exaustão o debate sobre as diversas correntes. A seguir, apresentam-se definições que servirão de enfoque para propor uma correlação de inovação tecnológica e comportamento do consumidor que servirá de adoção no presente estudo.

Sabe-se que o estudo do processo de inovação e seus efeitos estão diretamente associados a um setor específico da atividade econômica, ou seja, o setor industrial. Os estudos clássicos sobre inovação geralmente se referem às organizações que interagem com ambientes relativamente maduros e cujos produtos e tecnologias apresentam longos ciclos de vida. Esse é o caso de empresas pertencentes a setores industriais tradicionais, que não são significativamente afetados por revoluções tecnológicas ou por novas preferências de mercado.

\subsection{Inovação tecnológica}

Segundo Pavitt (1984), a discussão sobre a inovação tecnológica passa pela compreensão de uma taxonomia que descreve as firmas interagindo com a ciência, em que o progresso tecnológico se realiza por contato com instituiçóes de pesquisa, por gastos com $\mathrm{P} \& \mathrm{D}$ em laboratórios próprios e por aquisição de máquinas de fornecedores especializados. Essa diversidade de fontes do progresso tecnológico fornece a base para a compreensão do papel e do sentido dos fluxos tecnológicos entre as firmas e da interação entre elas. Essa formulação contribui para a compreensão das assimetrias entre as firmas.

Os trabalhos de Rosenberg (1976) e de Patel e Pavitt (1994) destacam o papel das inovações incrementais, da realização de melhoramentos e aperfeiçoamentos para o novo produto. Tais inovações incrementais são decisivas para definir o volume 
de vendas e o tempo de penetração de um novo produto em um novo mercado. De acordo com Rosenberg (1976), a imitação não é um processo passivo nem se reduz a uma cópia. Os trabalhos de Rosenberg e Pavitt enfatizam os elementos de continuidade no processo de inovação e sua relação com a competitividade.

A concorrência em termos dinâmicos assume uma condição específica para todo o sistema econômico, especificamente para as atividades de P\&D da firma. Como fator de motivação para que a empresa se empenhe em processos inovadores, o lucro se realiza por meio de assimetrias tecnológicas que permitem a ela apropriar-se de um lucro diferencial. Buscando reduzir o grau de incerteza, as firmas adotam comportamentos de rotina que servem como norteadores para as suas ações pautadas no aprendizado acumulado durante a sua história, possibilitando-lhe uma regularidade direcional das inovações.

A inovação tecnológica tem um lugar de destaque na teoria do desenvolvimento econômico preconizada por Schumpeter no início do século XX. De acordo com esse pressuposto, Rothwell (1995) descreve a inovação em vários aspectos, entre eles: a) introdução de um novo bem ou de uma nova qualidade de um certo bem; b) introdução de um novo método de produção; c) abertura de um novo mercado para uma indústria; d) utilização de uma nova fonte de matéria-prima ou produto semiacabado; e) estabelecimento de uma nova organização em uma determinada indústria.

Dosi (1988) retoma o conceito de Schumpeter ao afirmar que a inovação está ligada à busca e à descoberta, à experimentação e à adoção de novos produtos, aos processos de produção e a formas organizacionais. Continuamente, as empresas são estimuladas por clientes e pela própria concorrência a criar algo a ser apresentado, ou algum tipo de incremento que gere valor agregado a um produto já existente. De acordo com Robertson (1999), a inovação é produto de dois fatores principais: a necessidade e a adoção dos clientes e a competitividade do segmento. Essas duas forças atuam de forma positiva, gerando o chamado "efeito red queen", segundo o qual não se deve parar de avançar, mesmo sem sair do lugar, apenas para manter a mesma posição e não se deixar vencer pelos concorrentes.

Recorrendo, ainda, à ampla diversidade dos trabalhos sobre o tema, há duas formas distintas de inovação (FREEMAN; PEREZ, 1988; TUSHMAN; NADLER, 1997). Nessa perspectiva, a inovação tem sido usualmente classificada em incremental e radical. A inovação incremental refere-se à inovação linear, na qual um conceito básico é reforçado por melhorias contínuas, ou seja, busca-se o aperfeiçoamento de algo que já existe. Esse processo é denominado contínuo ou incremental quando trata 
apenas da adição de uma nova funcionalidade ao produto ou serviço, para que ele se torne mais atrativo para o mercado. As inovações contínuas referem-se a modificações ou extensões de produtos já existentes, o que não interfere de forma significativa no padrão básico do produto com o qual o consumidor já está acostumado.

Por sua vez, a inovação radical é entendida como uma inovação não linear, na qual emerge um conceito novo, causando uma ruptura com as práticas dominantes. Segundo Engel (2000), ela se configura como um produto totalmente novo, fato que altera significativamente os padrões de comportamento de consumo, pois é resultado de pesquisas específicas e deliberadas em laboratórios de Pesquisa e Desenvolvimento (P\&D), em universidades e em instituiçôes governamentais.

Quanto às inovaçôes tecnológicas, Christensen (1997) também define os produtos radicalmente inovadores como inovações descontínuas. Como exemplos disso, há computadores, televisores e telefones celulares. Da mesma forma, os produtos e serviços que mostram apenas incrementos a algo já existente são classificados por Robertson (1971) como inovações contínuas, cujos exemplos são os televisores digitais e os telefones com funções específicas. Há ainda uma terceira classificação, apresentada por Hoyer e MacInnis (2001), a qual se refere aos produtos que não são totalmente inovadores, mas representam algo além de um simples incremento Éas inovações dinamicamente contínuas, como, por exemplo, os notebooks e CDs em suas devidas épocas de lançamento.

Observa-se que, convencionalmente, a inovação está diretamente relacionada com o surgimento de novas tecnologias, sejam radicais ou apenas incrementais. Essa assertiva pode ser reforçada quando Grant (1998) afirma que a inovação é decorrente da evolução tecnológica. Ainda sobre esse ponto, Souza (2002) coloca o termo tecnologia como parte integrante da inovação, visto que, segundo o autor, "a tecnologia associa-se, muitas vezes, ao termo inovação; contudo, observa-se que este último é igualmente impreciso, sendo utilizado, indistintamente, para qualificar tanto 'novas tecnologias' quanto 'novas ideias'. Ou seja, para Souza (2002), inovação é um termo amplo que engloba diversos fatores novos, sejam eles quais forem, enquanto tecnologia é um ponto específico dentro desse escopo. É interessante colocar que o conceito de tecnologia pode alterar-se, de acordo com a época em que foi estabelecido, bem como os exemplos utilizados para tal definição, pelo rápido avanço das inovações.

Como tal, Nelson e Winter (1982) entendem por tecnologia o conjunto de ações que procura garantir a materialização de objetivos e a solução de problemas com base em uma determinada estrutura. Outra definição interessante é dada por 
Joerges (1988), para quem a tecnologia refere-se a máquinas modernas, tidas como coisas artificiais que desempenham uma grande quantidade de tarefas sozinhas e requerem conhecimento específico de engenharia e design para serem produzidas.

Nesse conceito podem ser inseridos ainda os serviços tecnológicos, desempenhados com o auxílio do homem e sua interação com hardwares e softwares. Para Rogers (1995), as ações voltadas para a produção de tecnologia são estruturadas por dois itens principais: hardware - sob um aspecto material; e software - relativo à informação enviada para o funcionamento da ferramenta. A interação entre hardware, software e ser humano seria, portanto, responsável pela produção e pela disseminação de produtos e serviços tecnológicos.

Outro aspecto dessa interação é descrito por Pavitt (1990) como a relação entre capacidade de inovação e inovação tecnológica, chamando a atenção para a interação entre as equipes de $\mathrm{P} \& \mathrm{D}$ e as demais áreas funcionais da empresa, assim como para as decisões estratégicas tomadas em cada uma delas, explorando o fluxo de comunicação dentro e entre as firmas. Nessa perspectiva, as pressões da concorrência dividem os esforços para a tomada de decisão em duas frentes: inovar para responder contínua e paulatinamente ao mercado ou focar em inovaçôes mais radicais, de alto grau de incerteza e tempo, mas potenciais para vantagens competitivas substanciais.

Quando se trata especificamente de serviços, devem-se levar em consideração as interaçōes apresentadas pelo modelo do Triângulo de Marketing de Serviços, desenvolvido por Kotler em 1994, em paralelo ao Conceito Piramidal, apresentado por Parasuraman (2000). A comparação entre esses dois modelos pode ser útil na visualização dos fenômenos interativos envolvendo diversos atores organizacionais com o advento da tecnologia.

No modelo triangular tradicional, observa-se a existência de três facetas: Marketing Externo, Marketing Interno e Marketing Interativo. A primeira faceta é a mais conhecida e explorada pelas organizaçôes, visto que trata da relação empresa-cliente. Essa relação pode ser interpretada como as promessas de atuação da empresa no mercado. Porém, de acordo com Parasuraman e Colby (2002), o relacionamento empresa-mercado pode ser seriamente comprometido caso não haja um bom desenvolvimento das ligaçôes relativas ao relacionamento empresa-funcionários e funcionários-clientes. Essa afirmação pode ser reforçada pelo fato de os serviços necessitarem da presença constante de pessoas para sua realização.

No caso dos serviços que utilizam tecnologia, considera-se uma quarta aresta do triângulo, que passa a ser tridimensional. Essa nova faceta é a tecnologia, que interage de forma igual com os três demais componentes do triângulo clássico. É 
importante salientar que o advento da tecnologia provocou mudanças para todos os atores que compõem o universo da organização. $\mathrm{O}$ modelo piramidal apresentado em Parasuraman (2000) torna clara a importância desse novo artifício e suas interferências nos novos modelos de gestão para serviços tecnológicos.

\subsection{Comportamento do consumidor e adoção de produtos e serviços de alta tecnologia}

Alinhados à perspectiva de Hawkins et al. (2001) e Solomon (1998), o estudo do comportamento do consumidor é definido como a área que avalia a forma pela qual indivíduos, grupos e organizações selecionam, compram, usam e dispõem de bens, serviços, ideias ou experiências para a satisfação de suas necessidades e desejos.

Para compreender melhor o atual modelo de comportamento de produtos de alta tecnologia, necessita-se observar a sua evolução nos últimos anos. Nesse sentido, Mowen e Minor (2003) conceituam o comportamento do consumidor como o estudo das unidades compradoras e dos processos de troca que estão envolvidos em adquirir, dispor e consumir produtos, serviços, experiências e ideias. Engel (2000) e Caro (2005) entendem o estudo do comportamento do consumidor como uma atividade voltada a compreender as tarefas envolvidas em obter, dispor e consumir produtos e serviços, incluindo-se nesse estudo os processos decisórios que sucedem e antecedem tais decisōes.

De acordo com Jeunon (2005), "independentemente do enfoque dos modelos, de forma geral, o comportamento do consumidor é visto como um processo de tomada de decisão influenciado por um conjunto de variáveis externas e internas". Engel (2000) complementa essa afirmação quando ressalta que a análise do comportamento do consumidor deve ser feita com base em fatores que exercem influência sobre o comportamento, como questôes pessoais e socioculturais.

Segundo Parasuraman e Colby (2002), existem assimetrias no comportamento do consumidor de produtos de alta tecnologia. No que se refere ao conceito de adoção de produtos e serviços de alta tecnologia, Rogers (2003) define as seguintes variáveis como fatores importantes para a avaliação do grau de adoção: (i) características percebidas de uma inovação, ou seja, vantagem relativa, compatibilidade, complexidade, "experimentabilidade" e "observabilidade"; (ii) vantagem relativa, que é o grau em que um indivíduo percebe uma inovação como sendo melhor do que aquela que a precedeu; (iii) compatibilidade, ou seja, grau em que um indivíduo percebe uma inovação como sendo consistente com os valores existentes, experiências 
passadas e necessidades dos adotantes; (iv) complexidade, que é o grau no qual um indivíduo percebe uma inovação como relativamente difícil de entender e usar; (v) experimentabilidade, isto é, o grau no qual um indivíduo pode experimentar uma inovação de um modo limitado, e (vi) observabilidade, que pode ser definida como o grau no qual os resultados de uma inovação podem ser observados por outros.

Em complemento à visão de Rogers (2003), Parasuraman e Colby (2002) acreditam existir quatro princípios que determinam o caráter exclusivo do perfil de consumidores de alta tecnologia, são eles: a) a adoção de tecnologia é um processo distinto; b) as inovações tecnológicas exigem estratégias de marketing diferenciadas; c) garantir a satisfação do cliente é um desafio maior para produtos e serviços baseados em tecnologia; d) os mercados de tecnologia são regidos pela lei da massa crítica em que, frequentemente, o resultado é do tipo "o vencedor fica com tudo" (PARASURAMAN; COLBY, 2002).

Parasuraman e Colby (2002) analisam cada um desses princípios como uma prática de marketing que parte do esforço aplicado em busca da compreensão de comportamento do consumidor de produtos high tech.

No decorrer dos estudos sobre o comportamento do consumidor de alta tecnologia, alguns autores buscaram estabelecer uma classificação em que os consumidores são segmentados por seus hábitos inovadores de consumo. Uma tentativa de classificação ocorreu em uma pesquisa, realizada pela companhia Data Forrester Research Inc., citada por Judge (1998) em seu artigo, na qual os consumidores de alta tecnologia são classificados em otimistas e pessimistas, considerando as atitudes dos indivíduos relativas à carreira, à família e aos momentos de lazer.

De acordo com tal classificação, existem duas dimensões básicas: otimistas e pessimistas. Como otimistas, estão classificados os consumidores: 1) acelerados: os que mais gastam e os primeiros a adotar tecnologias; 2 ) sustentadores da nova era: gastam muito, mas são focados em tecnologia de uso doméstico; 3) viciados em mouse: gostam de entretenimento on-line e estão dispostos a gastar para adquirir o que há de mais moderno; 4) techno batalhadores: os que utilizam a tecnologia para obter sucesso profissional; 5) esperançosos digitais: famílias com orçamentos limitados, mas interessadas em novas tecnologias; 6) apreciadores de gadgets: os que gostam de entretenimento on-line, mas dispõem de pouco dinheiro para gastar. Entre os pessimistas: 1) apertadores de mão: consumidores mais velhos que não tocam em seus computadores no trabalho; 2) tradicionalistas: dispostos a usar tecnologia, mas são lentos em sua adoção; 3) viciados em mídias: buscam entretenimento, mas não se encontram no mundo on-line. Observa-se, ainda, por meio dessa classificação, 
que nem sempre o otimismo é motivo suficiente para o consumo high tech. Fatores como recursos disponíveis e renda são determinantes para a efetiva atitude em direção à adoção de tecnologia.

Nesse sentido, segundo Rogers (1995), os consumidores podem ser segmentados, quanto à adoção da inovação, em: 1) Inovadores; 2) Adotantes iniciais; 3) Maioria inicial; 4) Maioria tardia; e 5) Retardatários. Classificação semelhante também é utilizada por Moore (1995), que introduz na teoria o conceito do abismo. Segundo o autor, há um gap entre os inovadores e adotantes iniciais e as outras duas classificações que, de alguma forma, adotam produtos, mesmo que tardiamente. Ainda segundo Moore (1995), o desafio das organizações é transpor essa lacuna e chegar até o consumidor.

Inseridos em uma proposta semelhante, Parasuraman e Colby (2002) também tentaram classificar os indivíduos segundo seus hábitos de consumo dentro do universo high tech, a saber: 1) Exploradores: são os primeiros a chegar, altamente motivados e sem medo; 2) Pioneiros: desejam os benefícios da nova terra, mas são mais práticos a respeito das dificuldades e dos perigos; 3) Céticos: precisam ser convencidos dos benefícios dessa nova fronteira; 4) Paranoicos: estão convencidos dos benefícios, mas são extraordinariamente preocupados com os riscos de se viver nesse novo contexto; 5) Retardatários: podem nunca vir a usar, a menos que sejam forçados a isso. Como pode ser observado, cada indivíduo possui peculiaridades no que se relaciona à adoção de produtos de alta tecnologia.

Nesse contexto, o construto Prontidão para Tecnologia, ou Technology Readiness (TR), refere-se à mensuração da propensão de os indivíduos adotarem tecnologias. De acordo com Parasuraman e Colby (2002), a TR apresenta algumas características específicas: (1) A TR varia de um indivíduo para outro - o percurso levado até a adoção da tecnologia depende do perfil de cada pessoa, o que resultará em TRs com graus de manifestação diversos; (2) A TR é multifacetada, ou seja, o grau da TR de uma pessoa é estabelecido de acordo com a mistura de diferentes crenças; (3) A TR presume e esclarece a resposta do consumidor de novas tecnologias, isto é, a TR busca prever e explicar o percentual de adoção de tecnologia, bem como sua forma de uso.

Ainda segundo os autores, as facetas negativas e positivas influenciam/contribuem para a atitude do consumidor em relação a produtos tecnológicos. Essas facetas estão relacionadas a sentimentos positivos, negativos e/ou neutros referentes à adoção de tecnologia. 
Cabe salientar que os sentimentos que envolvem o ser humano rumo à adoção, ou não, de tecnologia diferem das quatro dimensões estabelecidas por Parasuraman (2000) e Parasuraman e Colby (2002) para avaliação do grau de adoção, as quais são: otimismo, caráter inovador, desconforto e insegurança. Os autores descrevem cada dimensão detalhadamente, como pode ser observado a seguir:

- Otimismo: é uma dimensão definida como uma visão positiva da tecnologia e a crença de que ela propicia às pessoas maior controle, flexibilidade $\mathrm{e}$ eficiência nas suas vidas.

- Caráter inovador: tendência de um indivíduo ser pioneiro em tecnologia e líder de pensamento.

- Desconforto: falta de controle percebida sobre a tecnologia e o sentimento de ser oprimido por ela.

- Insegurança: refere-se a desconfiança e ceticismo a respeito da capacidade da tecnologia funcionar corretamente.

O modelo de dimensóes, criado por Parasuraman (2000); Parasuraman e Colby (2002), foi construído sob a consideração de dois tipos distintos de classificação: fatores inibidores (desconforto e insegurança) e fatores contribuintes (otimismo e caráter inovador) para a inovação, como pode ser observado na Figura 1.

FIGURA 1

Construto prontidão para tecnologia

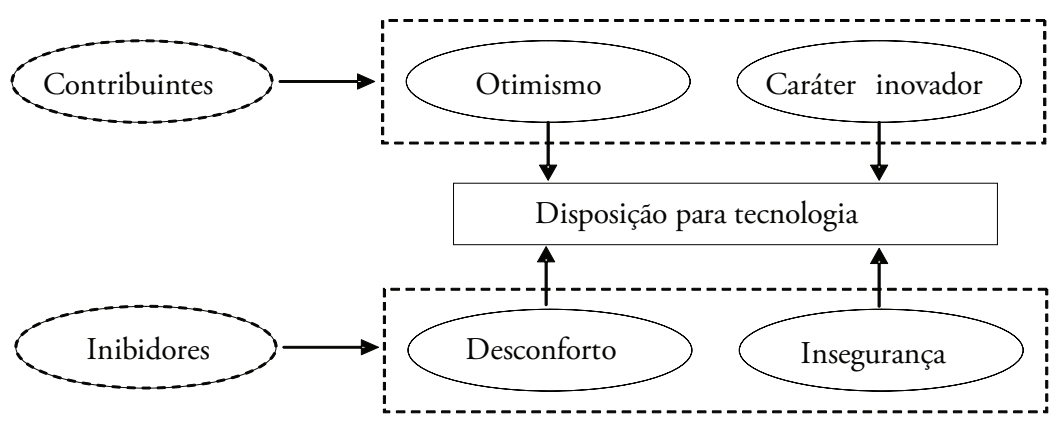

Fonte: Parasuraman e Colby (2002).

O processo de construção do Technology Readiness Index (TRI), ou Índice de Prontidão para Tecnologia, merece destaque nesta revisão bibliográfica a fim de se obter um índice confiável para aplicabilidade em contextos diversos. Destaca-se 
que a TRI foi elaborada com base em um programa de pesquisa multifásico, coordenado pelo professor da Universidade de Miami, A. Parasuraman, em parceria com Charles Colby, presidente da Rockbridges Associate.

Tsikriktsis (2004) promoveu um estudo de reaplicação da taxonomia proposta por Parasuraman e Colby (2001) em uma amostra no Reino Unido. Os resultados mostraram que apenas quatro dos cinco tipos de clientes se destacaram: explorador, pioneiro, cético e retardatário. $\mathrm{O}$ agrupamento paranoico não se evidenciou nos dados da pesquisa.

É interessante destacar que Souza (2002) realizou procedimento semelhante ao de Parasuraman (2000) no Brasil, tornando a escala válida também para utilização neste país.

\section{Procedimentos metodológicos}

Com a finalidade de buscar evidências empíricas à questão de pesquisa, o estudo realizado teve natureza aplicada, cunho exploratório, em uma primeira etapa, e descritivo, em uma segunda etapa. Segundo Cervo e Bervian (2002), uma pesquisa descritiva caracteriza-se por observar, registrar e correlacionar ocorrências, sem manipulação das variáveis. A presente pesquisa foi desenhada para aplicação de um survey transversal.

$\mathrm{O}$ universo pesquisado foi composto por consumidores e não consumidores de produtos eletrônicos de alta tecnologia na cidade de Maceió. A população da pesquisa foi estimada em 903.463 habitantes de Maceió (IBGE, 2005). A margem de erro da amostra foi determinada de acordo com um cálculo estatístico para populações finitas, apresentado por Malhotra (2001). Considerando um nível de confiança de $95 \%$ e 326 indivíduos pesquisados, a margem de erro calculada foi de $5,4 \%$.

A coleta de dados foi feita pelo método survey, com entrevistas pessoais, sendo o questionário preenchido pelo próprio entrevistador em $87,54 \%$ dos casos, e em $12,45 \%$ por e-mail nos meses de março a junho de 2007 . Os locais de coleta foram faculdades, empresas públicas e privadas e shoppings centers.

A pesquisa foi realizada com base no modelo de TRI criado por Parasuraman (2000). O instrumento aplicado aos consumidores do município de Maceió foi prétestado e adaptado às necessidades locais, sendo mantidas, no entanto, as principais características da escala. 
$\mathrm{O}$ instrumento ficou estruturado da seguinte forma:

- $1^{\text {a }}$ parte - Posse e uso de produtos/serviços tecnológicos: 19 questôes relativas a posse e pretensão/não pretensão de posse, bem como ao uso e pretensão/não pretensão de uso de produtos/serviços baseados em tecnologia. Nesse caso, sentiu-se a necessidade de um esforço de atualização dos itens tidos como inovações tecnológicas em detrimento das pesquisas anteriores.

- 2 $2^{\text {a }}$ parte - Escala TRI (Technology Readiness Index): composta por 36 afirmações sobre tecnologia, com respostas estruturadas em escalas com variação de 5 pontos, envolvendo o modelo das quatro dimensões (otimismo, "inovatividade", desconforto e insegurança) de Parasuraman (2000). Sendo dez questōes sobre otimismo, sete sobre "inovatividade", dez sobre desconforto e nove sobre insegurança.

- $3^{a}$ parte - Caracterização dos entrevistados: onze questôes relacionadas ao perfil dos respondentes. As variáveis eram sexo, faixa etária, estado civil, grau de instrução, ocupação, renda familiar, quantidade de pessoas que moram com o entrevistado, quantidade de pessoas que moram com o entrevistado e utilizam tecnologia, uso de tecnologia no trabalho, naturalidade, local onde reside atualmente. É válido salientar que, para a presente pesquisa, só foram exploradas as variáveis do perfil que se adequaram às análises realizadas.

Inicialmente, para a determinação dos itens considerados eletrônicos de alta tecnologia (gadgets eletrônicos), foi feita uma pesquisa exploratória, via internet, com a finalidade de obter o grau de conhecimento relativo a esses produtos, com indivíduos de idade e perfil (a priori) equivalentes ao público-alvo da pesquisa. De acordo com os resultados obtidos, gadgets citados com maior relevância foram adotados como elementos centrais a serem trabalhados na pesquisa, a saber: black berry, mp4 player (ipod), lap top, pen drive, mp3 player, palm top, TV de plasma, gravador de DVD, câmera fotográfica, filmadora digital e videogame.

Para a tabulação e análise dos dados foi utilizado o software de análise estatística SPSS (Statistic Package for Social Science), o qual possibilitou o desempenho de análises univariadas e multivariadas.

Foram feitas análises descritivas (frequência, média e desvio-padrão), testes de hipóteses (teste $t$ e análise de variância) e uma análise multivariada (Análise de Agrupamentos - AA). 
Luciana Peixoto Santa Rita, Alcides Carlos de Araújo, Mainah Almeida de Paula, Manuella de Oliveira Lima, José Carlos Viana Filho

Com base na literatura estudada, foram formuladas duas hipóteses para testar o argumento de Parasuraman (2000), que diz o seguinte: quanto maior o Índice de Prontidão para a Tecnologia (TRI), maior a receptividade a ela, como:

- $\mathrm{Ha}_{1}$ : Entrevistados que utilizam tecnologia no trabalho possuem média de TRI maior que as pessoas que não utilizam.

- $\mathrm{Ha}_{2}$ : Entrevistados que moram com um número maior de pessoas que utilizam tecnologia tendem a possuir maior TRI em relação às pessoas que moram com menor número de pessoas.

Para análises inferenciais acerca das hipóteses supracitadas, foram utilizados testes t e análise de variância de um critério (one-way analysis of variance - one way ANOVA) com o complemento do teste Tukey. Além disso, para averiguaçōes sobre o comportamento da amostra, utilizou-se a técnica de estimativas com um intervalo de confiança de $95 \%$.

Com o intuito de descobrir e classificar os tipos de clientes de alta tecnologia uma análise de agrupamentos foi realizada. Seguindo as orientaçôes de Hair Junior et al. (2005), ela foi executada em seis estágios que estão citados abaixo.

No primeiro foram definidos os objetivos da análise, que se traduzem em buscar confirmação sobre os tipos de clientes encontrados por Parasuraman e Colby (2002), e selecionar as variáveis de agrupamento que foram as facetas da TRI. No segundo houve a definição da medida de similaridade (neste caso medida de distância euclideana quadrada) e, sendo necessária, a padronização dos dados. Verificou-se que não era preciso padronizar os dados, uma vez que todas as variáveis usadas em análise apresentavam a mesma escala. $\mathrm{Na}$ terceira etapa, buscou-se atender às suposiçôes da análise de agrupamento: a representatividade da amostra e multicolinearidade envolvida. Em seguida, foram determinados os agrupamentos e a avaliação do ajuste geral. Isso ocorreu por meio dos métodos: hierárquico, pelo método de aglomeração ward, que serviu para orientar sobre os centros de agrupamentos e detecção de observaçōes atípicas por um esquema de aglomeração obtido na análise (em que é possível verificar quais observações se agruparam tarde demais), e não hierárquico de agrupamentos, para determinar a solução final.

$\mathrm{Na}$ interpretação dos agrupamentos, quinta etapa, os grupos resultantes foram rotulados de acordo com a variável estatística. Por fim, na última etapa, houve o esforço para a validação dos agrupamentos por meio de aplicação de um método alternativo não hierárquico com pontos sementes aleatórios (em vez de pré-especi- 
ficados). Os pontos sementes são centros de agrupamento ou valores designados a cada variável de agrupamento (HAIR JUNIOR, 2005).

\section{Resultados e discussão}

Esta seção apresenta os dados relativos às dimensões e aos fatores do estudo. Em um primeiro momento, a análise individual dos fatores tem como eixo o conteúdo em si, sem a exigência de uma análise do problema e suas devidas associaçôes. Por meio desse procedimento, procura-se caracterizar o perfil dos respondentes no primeiro bloco do questionário. Para isso, utilizou-se frequência que demonstrará algumas classificações. Em seguida, serão mostradas as associações encontradas na situação pesquisada. Pelas limitações do estudo, esses resultados precisam ser analisados à luz da teoria. A seguir, deve-se apresentar e comentar as relaçóes com o suporte da base estatística sem grande concentração em análises individuais, mas em um contexto que permita relacionar a pesquisa a outras pesquisas já desenvolvidas e abrir novas vertentes para futuras investigaçōes.

\subsection{Perfil dos pesquisados e média da TRI}

A amostra válida da pesquisa foi composta por 326 questionários, nos quais se verificou predomínio do sexo masculino $(57,1 \%)$. Um índice de 58\% da amostra caracterizou-se pela faixa de "16 a 23 anos" e $72,4 \%$, pela de solteiros. A renda familiar foi bem distribuída, com predominância de "1 a 5 salários" (33,7\%), e em seguida de "mais de 5 a 10 salários" (29,8\%). Quanto à ocupação, a maioria dos respondentes era estudantes $(50,9 \%)$ seguidos de funcionários de empresa privada $(27,9 \%)$. Em relação ao grau de instrução, aproximadamente $51,5 \%$ tinham superior incompleto; $21,2 \%$, ensino médio completo; e $14,1 \%$, superior completo. A maior parte dos entrevistados afirmou utilizar tecnologia em seu trabalho $(73,3 \%)$.

O cálculo do Índice de Prontidão para Tecnologia foi feito segundo orientações de Parasuraman (2000) e Tsikriktsis (2004). Parasuraman (2000, p. 13) apenas orienta intuitivamente sobre a fórmula, lembrando que é necessária uma codificação reversa para os escores das dimensões Desconforto e Insegurança, enquanto em Tsikriktsis (2004) vemos a fórmula propriamente dita. É simplesmente uma média entre as quatro facetas da TRI, mas considerando que as dimensões Otimismo e Inovatividade são contribuintes do índice e as dimensões Desconforto e Insegurança são inibidoras, temos a seguinte fórmula: 
Luciana Peixoto Santa Rita, Alcides Carlos de Araújo, Mainah Almeida de Paula, Manuella de Oliveira Lima, José Carlos Viana Filho

$$
T R I=\frac{\text { Otim. }+ \text { Inov. }+(6-\text { Desc. })+(6-\text { Inseg. })}{4}
$$

A média do Índice de Prontidão para a Tecnologia (TRI) encontrado por meio da amostra foi de 3,02. Da classificação das médias das dimensões têm-se aproximadamente 3,81 para Otimismo, 3,24 para Inovatividade, 3,30 para Desconforto e 3,65 para Insegurança. A Tabela 1 demonstra as médias dos fatores do TRI em outros estudos. Segundo Parasuraman (2000), quanto maior o Índice de Prontidão para a Tecnologia, maior a receptividade e a adoção de produtos e serviços de alta tecnologia.

TABELA 1

Comparação dos componentes do Índice de Prontidão para a Tecnologia (TRI)

\begin{tabular}{lccc}
\hline Componentes & Parasuraman (2000) & Tsikriktsis (2004) & Pesquisa \\
\hline Otimismo & 3,84 & 3,60 & 3,81 \\
Inovatividade & 3,18 & 3,23 & 3,24 \\
Desconforto & 3,46 & 3,54 & 3,30 \\
Insegurança & 4,03 & 3,82 & 3,65 \\
\hline
\end{tabular}

Fonte: Parasuraman (2000), Tsikriktsis (2004) e dados da pesquisa.

Como entrevistamos parte dos consumidores face a face e parte por e-mail, buscou-se analisar a média do TRI para esses indivíduos a fim de verificar existência de vieses nos métodos de coleta de dados. Os resultados são visualizados abaixo.

TABELA 2

Comparação da coleta de dados

\begin{tabular}{l|c|c|c|c|c}
\hline $\begin{array}{l}\text { Tipo de coleta } \\
\text { de dados }\end{array}$ & Frequência & Média & DP & t & p \\
\hline E-mail & 41 & 2,97 & 0,357 & $-0,89$ & 0,374 \\
Face a face & 285 & 3,02 & 0,359 & & \\
\hline
\end{tabular}

Fonte: Dados da pesquisa.

Existiriam diferenças significativas caso o valor de $p<0,05$. Contudo, diante dos resultados da Tabela 2 , demonstramos não existirem diferenças significativas entre os tipos de coleta de dados. 


\subsection{Intervalos de confiança}

As dimensōes foram caracterizadas pela construção de intervalos de confiança ao nível de $95 \%$. Isso permitiu encontrar os limites máximos e os mínimos para o perfil dos usuários abordados na pesquisa. Os principais resultados estão expostos na Tabela 3.

TABELA 3

Intervalos de confiança

\begin{tabular}{l|c}
\hline Tópico verificado & Intervalo de confiança \\
\hline Proporção de pessoas que utilizam tecnologia no trabalho & $71 \%$ e $81 \%$ \\
Média da TRI & 2,99 e 3,09 \\
Dimensão otimismo & 3,75 e 3,89 \\
Dimensão inovatividade & 3,14 e 3,28 \\
Dimensão desconforto & 3,17 e 3,31 \\
Dimensão insegurança & 3,56 e 3,73 \\
\hline
\end{tabular}

Fonte: Dados da pesquisa.

Observa-se que os maiores valores nos intervalos foram encontrados na dimensão otimismo. Nessa direção, entende-se que esses consumidores são os que mais gastam e os primeiros a adotar novas tecnologias para uso pessoal.

\subsection{Testes das hipóteses}

Buscou-se testar hipóteses que identificassem características específicas de consumidores de tecnologia. Para tanto, foram realizadas medidas para a confirmação de duas hipóteses geradas pela pesquisa. Quanto à hipótese $\mathrm{Ha}_{1}$, utilizamos o teste $t$ como instrumento de análise. Para a suposição $\mathrm{Ha}_{2}$, fizemos o teste ANOVA com o complemento do teste Tukey.

Antes das análises, buscou-se avaliar as suposições exigidas pelos testes. São elas: a) independência entre as respostas dos entrevistados; b) normalidade, a variável explicada (TRI) apresenta distribuição em "forma de sino" para cada grupo e c) homocedasticidade, as variâncias da variável dependente são estatisticamente iguais em todos os grupos (HAIR JUNIOR et al., 2005). Leech, Barret e Morgan (2005) citam que todos os testes estatísticos possuem suas suposiçōes, as quais demonstram 
se realmente a medida de análise está sendo desenvolvida corretamente. Hair Junior et al. (2005) aconselham esse exame nos dados, pois ignorar tais cuidados pode conduzir a avaliações pouco precisas. Diante disso, foram feitos testes de aderência à normalidade (Kolmogorov-Smirnov) e de homogeneidade de variância (teste de Levene).

A estatística Kolmorogov-Smirnov demonstra o nível de significância em um teste de aderência, ou seja, se os dados observados possuem uma distribuição próxima de uma determinada distribuição esperada. Nesse caso, verificou-se se a variável TRI estava aproximadamente seguindo uma distribuição normal. A análise apresentou os seguintes resultados: K-S $=0,045$ e $p=0,199$. Determinou-se um nível crítico de 0,05 para valores $p$ abaixo de 0,05 para rejeitarmos a suposição de normalidade. Sendo assim, não poderíamos realizar os testes $t$ e ANOVA. Contudo, para um valor de $p=0,199$, aceitou-se a suposição de normalidade e continuaram as análises. Quanto à suposição de independência, buscou-se fazer as entrevistas individualmente com cada consumidor, logo, respeitou-se a proposição de independência entre os entrevistados. Em relação ao teste de Levene, abordaremos seus resultados tanto no teste $t$ quanto no teste ANOVA; dado que no primeiro observou-se a homogeneidade de variância para dois grupos, enquanto no segundo avaliou-se para cinco grupos.

Após esse relato sobre as suposições das estatísticas utilizadas neste estudo, convém destacar a primeira hipótese. $\mathrm{Ha}_{1}$ : Entrevistados que utilizam tecnologia no trabalho possuem média de TRI maior que as pessoas que não utilizam. Para testar essa hipótese foi realizado um teste $t$ para dois grupos independentes, monocaudal direito, com nível de significância de 0,05. Segundo Leech, Barret e Morgan (2004), a estatística $t$ é apropriada quando estudamos diferenças entre dois grupos. Os resultados são visualizados na tabela abaixo.

\begin{tabular}{l|c|c|c|c|c}
\multicolumn{7}{c}{$\begin{array}{c}\text { TABELA 4 } \\
\text { Teste t }\end{array}$} \\
$\begin{array}{l}\text { Utiliza tecnologia no } \\
\text { trabalho }\end{array}$ & Frequência & Média & DP & $\mathbf{t}$ & P \\
\hline Sim & 239 & 3,06 & 0,36 & 3,117 & $0,002(1)$ \\
Não & 85 & 2,92 & 0,31 & & \\
\hline
\end{tabular}

Fonte: Dados da pesquisa.

(1) Diferenças significantes ao nível de 0,05.

Nota: Teste de Levene: 2,$561 ; p=0,111$

Por meio do teste de Levene, verificou-se a suposição de homogeneidade de variâncias. Segundo Leech, Barret e Morgan (2004) e Hair Junior et al. (2005), deve-se aceitar a hipótese do teste de Levene (com nível de confiança de 0,05 ) 
quando obtemos valores $p$ acima de 0,05 . Logo, para um valor $p=0,111$, atendeuse à suposição. Dessa forma, os resultados obtidos no estudo demonstraram uma melhor confiabilidade. Ao observar a Tabela 3 acima, percebemos uma média maior da TRI no grupo que utiliza tecnologia no trabalho em relação ao que a não utiliza. Assim, foi feito o teste $t$ para verificarmos se as médias desses grupos eram diferentes estatisticamente. Foi obtido um $t=3,117$ e $p=0,002$ e podem-se evidenciar diferenças significativas entre os consumidores que utilizam tecnologia no trabalho e aqueles que a não utilizam. Nesse caso, maior grau de disposição à tecnologia para o primeiro grupo, e pode-se aceitar a hipótese $\mathrm{Ha}_{1}$.

A segunda hipótese propunha a seguinte afirmação: $\mathrm{Ha}_{2}$ : Entrevistados que moram com uma proporção maior de pessoas que utilizam tecnologia tendem a possuir maior TRI em relação às pessoas que moram com menor proporção de pessoas.

Para análise dessa proposição, a princípio foram estabelecidas classes e suas respectivas médias e desvios. Primeiramente, dividiu-se o número de pessoas que moram com o respondente e utilizam tecnologia pelo número de pessoas que moram com o respondente. Pode-se observar na fórmula abaixo como encontrar a proporção da família dos respondentes que utilizam tecnologia.

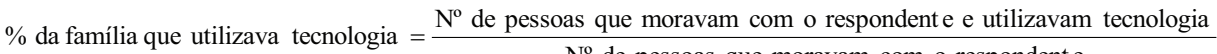
$\mathrm{N}^{\mathrm{o}}$ de pessoas que moravam com o respondente

Verificou-se a existência de seis missing values, devido ao fato de alguns indivíduos morarem sozinhos ou não possuírem família que utilizam tecnologia. Em seguida, estabeleceram-se cinco categorias para formação dos grupos. Os resultados podem ser demonstrados na tabela abaixo.

TABELA 5

Proporção de pessoas que moram com o respondente e utilizam tecnologia

\begin{tabular}{lcccc}
\hline Classes & Frequência & Média TRI & Desvio-padrão TRI \\
\hline $0 \%-20 \%$ & 78 & 2,91 & 0,32 \\
$20 \%-40 \%$ & 68 & 2,99 & 0,31 \\
$40 \%-60 \%$ & 63 & 3,06 & 0,37 \\
$60 \%-80 \%$ & 40 & 3,04 & 0,40 \\
$80 \%-100 \%$ & 71 & 3,09 & 0,33 \\
\hline
\end{tabular}

Fonte: Dados da pesquisa.

Nota: Teste de Levene: 1,$543 ; p=0,190$ 
No teste de Levene avaliou-se a suposição de homogeneidade de variâncias para cinco grupos. O resultado aponta para a aceitação da homocedasticidade ( $p$ $=0,190$, ou seja, $p>0,05)$. Dessa forma, pode-se realizar o teste ANOVA com melhor confiabilidade e precisão. Além disso, pode-se observar, na Tabela 4, como as médias da TRI nos grupos aumentaram à medida que aumentava a proporção de pessoas que moravam com o respondente e utilizavam tecnologia. Somente na classe 60\%-80\% houve um decréscimo na média, mas voltando a crescer na classe posterior. A importância da análise de variância ocorreu em razão da necessidade de verificar se as médias desses grupos eram estatisticamente diferentes. Leech, Barret e Morgan (2004) apresentam o uso da referida análise quando o objetivo é comparar mais de dois grupos, nesse caso cinco grupos. Os resultados são visualizados abaixo.

TABELA 6

Teste ANOVA

\begin{tabular}{l|c|c|c|c|c}
\hline Variação & $\begin{array}{c}\text { Soma dos } \\
\text { quadrados }\end{array}$ & g.l. & $\begin{array}{c}\text { Quadrados } \\
\text { médios }\end{array}$ & $\boldsymbol{F}$ & $\boldsymbol{p}$ \\
\hline Entre grupos & 1,346 & 4 & 0,337 & 2,847 & 0,024 \\
Dentro dos grupos & 37,234 & 315 & 0,118 & & \\
Total & 38,580 & 319 & & & \\
\hline
\end{tabular}

Fonte: Dados da pesquisa.

Os resultados da tabela $(p=0,024$, ou seja, $p<0,05)$ apontam para a existência de diferenças significativas entre os grupos. Contudo, deve-se notar que o teste não apresenta quais são os grupos. Desse modo, complementa-se a análise de variância com o teste de Tukey, importante para conhecermos quais dos grupos apresentaram as diferenças significantes (LEECH; BARRET; MORGAN, 2004). A seguir, pode ser observada na Tabela 7 uma matriz de distâncias resumindo os resultados da análise.

De forma geral, pode-se observar que a maior distância estatisticamente significante está entre o grupo $0 \%-20 \%$ e o grupo $80 \%-100 \%$. Desse modo, aceita-se a hipótese $\mathrm{Ha}_{2}$, ou seja, os consumidores com maior propensão a tecnologia moram com pessoas também adeptas às tecnologias. Vale ressaltar que as diferenças foram significativas nos grupos extremos, ou seja, para que os familiares possam induzir a adoção de tecnologia no indivíduo é necessária uma proporção expressiva, isto é, entre $80 \%$ e $100 \%$ das pessoas. 
TABELA 7

Teste Tukey

\begin{tabular}{|c|c|c|c|c|c|}
\hline Distâncias & $0 \%-20 \%$ & $20 \%-40 \%$ & $40 \%-60 \%$ & $60 \%-80 \%$ & $80 \%-100 \%$ \\
\hline $0 \%-20 \%$ & 0 & $0,080(0,629)$ & $0,142(0,110)$ & $0,129(0,305)$ & $\begin{array}{c}0,175(0,018) \\
(1)\end{array}$ \\
\hline $20 \%-40 \%$ & & 0 & $0,062(0,843)$ & $0,049(0,953)$ & $0,095(0,481)$ \\
\hline $40 \%-60 \%$ & & & 0 & $0,013(0,999)$ & $0,033(0,981)$ \\
\hline $60 \%-80 \%$ & & & & 0 & $0,046(0,962)$ \\
\hline $80 \%-100 \%$ & & & & & 0 \\
\hline
\end{tabular}

Fonte: Dados da pesquisa.

(1) Diferenças de médias significantes ao nível de 0,05.

Nota: Valores $p$ entre parênteses.

\subsection{Análise de Agrupamentos (AA)}

Um dos passos iniciais em análise de agrupamento necessita da especificação da quantidade de agrupamento a ser gerada (HAIR JUNIOR et al., 2005). Para estudos de fins confirmatórios (como é o caso), a quantidade de agrupamentos depende do modelo que se está tentando confirmar. Por meio da análise proposta foram testados dois modelos, um com quatro e outro com cinco agrupamentos, quantidade a ser testada em cada modelo e observada em Parasuraman e Colby (2002), que apresenta um modelo de cinco grupos, enquanto Tsikriktsis (2004) aborda um modelo com quatro grupos.

Os resultados do modelo de cinco agrupamentos revelaram os mesmos cinco tipos de clientes encontrados por Parasuraman e Colby (2002): explorador, pioneiro, cético, paranoico e retardatário. Já o modelo de quatro agrupamentos encontrou os mesmos revelados por Tsikriktsis (2004), que não apresenta o agrupamento "paranoico". É importante ressaltar que os resultados do modelo de quatro agrupamentos não pretendiam encontrar o mesmo resultado de Tsikriktsis (2004), e qualquer um dos cinco tipos de clientes poderia ser excluído.

No entanto, os agrupamentos deveriam ser validados para que pudessem ter confiabilidade. A representatividade estava garantida, uma vez que constituía consumidores e não consumidores de produtos e serviços tecnológicos da cidade de Maceió - Alagoas. A confiabilidade foi testada por meio da aplicação de um método alternativo a cada agrupamento, esperando obter os mesmos resultados para passar na validação (HAIR JUNIOR et al., 2005). Como se conseguiu os agrupamentos 
pela análise não hierárquica com pontos sementes definidos, decidiu-se utilizar uma análise não hierárquica com pontos sementes aleatórios.

Dessa forma, somente o modelo com quatro agrupamentos foi validado, tendo o modelo de cinco agrupamentos omitido o tipo "paranoico" no processo. Importante lembrar que esse mesmo tipo não se encontra no modelo de quatro agrupamentos nem nos resultados de Tsikriktsis (2004). O modelo validado possui os seguintes tipos de clientes (e suas respectivas porções da amostra de 326 observações): explorador $(22,39 \%)$, pioneiro $(29,14 \%)$, cético $(17,18 \%)$ e retardatário $(31,29 \%)$.

No processo foram eliminadas 11 observações atípicas com base em um esquema de aglomeração obtido no método ward de análise hierárquica.

A seguir é demonstrado o perfil de cada agrupamento do modelo validado:

- Perfil - Exploradores: 68,5\% são do sexo masculino; 52,1\% têm de 16 a 23 anos; 75,3\% são solteiros; 28,8\% possuem renda familiar de mais de 10 a 15 salários mínimos; 65,8\% são naturais de Maceió; 81,9\% utilizam tecnologia no trabalho; e 45,2\% moram em bairros de classe B. Em relação ao percentual de familiares que utilizam/compram tecnologia regularmente, 29,4\% afirmaram que de 76 a 100\% de seus familiares são adotantes assíduos de tecnologia.

- Perfil - Pioneiros: $75,8 \%$ são homens; 53,7\% têm de 16 a 23 anos; 71,6\% são solteiros; $34,7 \%$ têm renda familiar de 1 a 5 salários mínimos; $56,8 \%$ são naturais de Maceió; $81,1 \%$ utilizam tecnologia no trabalho; $36,8 \%$ moram em bairros de classe C e 31,6\% afirmaram que de 26 a 50\% de seus familiares utilizam/compram tecnologia regularmente.

- Perfil - Céticos: 62,5\% são do sexo feminino; 66,1\% têm de 16 a 23 anos; $71,4 \%$ são solteiros; $42,9 \%$ possuem renda de 1 a 5 salários; $66,1 \%$ são naturais de Maceió; 67,3\% utilizam tecnologia no trabalho; 42,9\% moram em bairros de classe C; e 36,4\% afirmaram que de 26 a 50\% de seus familiares utilizam/compram tecnologia regularmente.

- Perfil - Retardatários: $57,8 \%$ são mulheres; $61,8 \%$ têm de 16 a 23 anos; $71,6 \%$ são solteiros; $40,2 \%$ possuem renda de 1 a 5 salários mínimos; $74,5 \%$ são naturais de Maceió; 64,7\% utilizam tecnologia no trabalho; $42,2 \%$ moram em bairros de classe C e 31,4\% afirmaram que de 26 a $50 \%$ de seus familiares são adotantes assíduos de produtos/serviços tecnológicos. 


\section{Considerações finais}

O estudo teve características peculiares, uma vez que se analisou o processo de adoção de compra de produtos e serviços eletrônicos de alta tecnologia por meio da Technology Readiness Index (TRI).

Para atingir esse objetivo, o estudo baseou-se nas pesquisas realizadas por Parasuraman (2000) e Parasuraman e Colby (2002), os quais desenvolveram a Technology Readines. Assim, foram formuladas duas hipóteses, aceitas para explicar comportamentos de posse e uso de produtos e serviços de alta tecnologia. Para efeito de modelo de análise, assumiu-se a frequência de cada categoria de resposta sobre posse e uso de produtos/serviços de alta tecnologia.

A primeira hipótese testou se as pessoas que utilizavam tecnologia no trabalho possuíam média mais alta de TRI que as pessoas que não utilizavam. Nessa direção, as inferências estatísticas revelaram que $(p=0,002<p=0,05)$, obtendo maior probabilidade de aceitação da hipótese de média mais alta dessas pessoas.

Por sua vez, a segunda hipótese testou se uma das médias da TRI era significativamente diferente das outras, implicando um maior índice de adoção de tecnologia por parte dos entrevistados que moram com pessoas que utilizam tecnologia. Os testes revelaram que ( $p=0,024<p=0,05)$, rejeitando-se a hipótese nula, ou seja, a proporção de pessoas que moram com o respondente e utilizam tecnologia afeta significativamente, a um nível de significância de 0,05 , a Prontidão para a Tecnologia (TRI).

A pesquisa de campo revelou, ainda, que a média do Índice de Prontidão para a Tecnologia (TRI) da amostra foi 3,02, sendo superior à de outros estudos. Da classificação das médias das dimensões, tem-se aproximadamente 3,81 para Otimismo, 3,24 para Inovatividade, 3,30 para Desconforto e 3,65 para Insegurança. De acordo com Parasuraman (2000), quanto maior o Índice de Prontidão para a Tecnologia (TRI), maior a receptividade. Os resultados apontam ainda o fato de a categoria Inovatividade, identificada como a tendência de um indivíduo ser pioneiro em tecnologia e líder de pensamento, ter apresentado a maior contribuição à agregação do índice. Analisando a variável sexo com a TRI, observa-se que o sexo masculino apresenta média de 3,11, demonstrando, assim, maior disposição para a tecnologia que o sexo feminino com 2,95, sendo também as maiores médias nas dimensões Otimismo e Inovatividade. Em relação aos entrevistados por e-mail e pessoalmente, os primeiros registraram média de TRI de 2,97, enquanto no que se refere aos entrevistados face a face encontramos média de 3,02. Baseando-se no teste $t$, tais valores não apresentaram diferenças significativas. 
Os resultados da análise de agrupamento demonstraram que a amostra pesquisada apresenta uma maior quantidade de retardatários (31,29\%). Isso revelou uma tendência negativa para a adoção de tecnologia, pois segundo Parasuraman e Colby (2002), os retardatários são os últimos a adotarem tecnologia, e só o fazem em momentos em que são "forçados" a isso, por exemplo, comprar um telefone celular para não ser excluído da vida em sociedade.

No entanto, os dados apresentam uma quantia considerável de pioneiros $(29,14 \%)$, que são, segundo Parasuraman e Colby (2002), os segundos a utilizarem tecnologia, logo depois os exploradores (22,39\%). Os céticos, que não se sentem inseguros ou desconfortáveis em adotar tecnologia, mas precisam ser convencidos de seus benefícios, possuem a menor parcela da amostra $(17,18 \%)$.

Uma apreciação relevante da análise dos resultados é o fato de a categoria "paranoicos" não ter representatividade nos resultados (TSIKRIKTSIS, 2004). Na pesquisa, observou-se que o envolvimento dos consumidores de alta tecnologia foi reduzido, o que pode comprometer o desenvolvimento das organizaçóes que atuam nessa área, surgindo, então, a necessidade de investimentos em estratégias tecnológicas.

De modo geral, o desenho prático desse estudo considerou que a adoção de produtos e serviços de alta tecnologia possui uma grande importância no cenário econômico mundial, não só pela temática da inovação, mas também pela discussão da correlação da gestão mercadológica e a categoria de produtos e serviços inovadores. Por esse prisma, as contribuiçóes, à luz da teoria e da escala adotada, trazem implicaçôes que delineiam a competitividade no processo de desenvolvimento de novos produtos e serviços.

No âmbito teórico, a relevância do estudo teve impacto sob duas vertentes: primeiro, com relação à produção nacional de pesquisa (básica e aplicada); e segundo, com relação ao fluxo e à difusão intersetorial de tecnologia no ambiente do marketing. Nesse sentido, as correlaçôes estabelecidas no modelo conceitual possibilitarão novas linhas de pesquisa. Ainda com a contribuição teórico/metodológica buscou-se evidenciar a comprovação de um modelo que permitiu fazer inferências correlacionais sobre o fenômeno.

Outro ponto para o reconhecimento acadêmico do estudo foi identificado com base nos estudos sobre comportamento do consumidor que se referem tão somente aos aspectos específicos dos produtos e serviços convencionais. A presente pesquisa assegurou o ineditismo, à luz dos medidores do marketing para produtos e serviços inovadores, que permitirá fomentar novos modelos de gestão, pesquisa e difusão de tecnologia. 
Nessa direção, um aspecto que até então inibia o avanço dessas pesquisas na área era a reduzida preocupação, tanto dos pesquisadores quanto dos órgãos envolvidos nesse campo, em organizar sistematicamente esses estudos apenas descrevendo a visão técnica da inovação.

Do lado da iniciativa privada, empresários e investidores devem estabelecer uma "rede relacional" que assume um significado mais amplo, envolvendo prestadores, fornecedores, clientes, centros de formação técnica, prestadores de serviços, agentes governamentais, instituiçóes financeiras, instituiçôes de ensino e pesquisa, todos articulados como cadeia e sistema de valor, principalmente pela necessidade de compreender a inovação aliada a um processo de gestão mercadológica.

Em especial, os dirigentes brasileiros se beneficiarão da análise dessa pesquisa, pois traz, espera-se, informações e discussões que, de um lado, ajudam a avaliar as mudanças em curso e, de outro, apontam um significativo impacto das práticas que se associam à disposição de consumo em produtos e serviços inovadores.

Como contribuição adicional à academia, a pesquisa descreveu uma extensa revisão e aplicação prática em relação ao fenômeno do comportamento de consumidor de produtos de alta tecnologia. Tais resultados podem auxiliar um melhor gerenciamento da relação cliente-tecnologia e consequente customização das estratégias de tecnologia das organizaçóes.

Em comparação com os estudos de Parasuraman e Colby (2002) e Tsikriktsis (2004), observa-se que a concentração desses segmentos se altera entre diversas localidades. Nessa direção, são necessárias ações de marketing diferenciadas dependendo do país em questão. Como no estudo apresentado por Tsikriktsis (2004), a amostra de pesquisa também não evidenciou o segmento paranoico. Porém, diferenças importantes foram encontradas na composição de cada agrupamento, talvez influenciadas pela cultura do local onde a pesquisa foi aplicada. Na presente pesquisa, por exemplo, o maior agrupamento foi constituído de retardatários (31\%), seguidos dos pioneiros (29\%), indicando que a maioria só usará tecnologia se alguma circunstância a forçar a isso. No entanto, o fato de os pioneiros serem o segundo maior agrupamento indica que há um mercado para tecnologia e ele pode crescer. $\mathrm{O}$ estudo de Tsikriktsis (2004) foi aplicado no Reino Unido, e o maior agrupamento identificado foi de pioneiros (32\%) seguidos dos exploradores (27\%), o que indica uma cultura mais voltada para a tecnologia. No estudo de Parasuraman e Colby (2002), o maior agrupamento foi de pioneiros (27\%), mas seguido de céticos (21\%) e paranoicos $(20 \%)$.

Existem diferenças também quanto ao perfil de cada agrupamento. No presente estudo, grande parte dos retardatários era do sexo feminino, com baixa renda, fatores 
idênticos aos resultados de Tsikriktsis (2004) e Parasuraman e Colby (2002). Por sua vez, os pioneiros são predominantemente masculinos, com renda baixa. No estudo de Tsikriktsis (2004) a maioria era do sexo feminino e de renda média, enquanto em Parasuraman e Colby (2002) a maior parte era do sexo masculino e de renda média. Os exploradores são na maioria do sexo masculino e de renda mediana. Da mesma forma que Tsikriktsis (2004), o estudo de Parasuraman e Colby (2002) identificou exploradores na maioria do sexo masculino e de renda alta. Finalmente, os céticos são na maioria do sexo feminino, de renda baixa. Tsikriktsis (2004) e Parasuraman e Colby (2002) registraram céticos como sendo do sexo masculino e de renda média. Novos estudos devem focar na comparação de tais diferenças entre várias regiōes e tentar identificar fatores que ajudem a explicá-las.

Assim, assumindo esses resultados, torna-se necessário destacar as seguintes limitações: a) os resultados da pesquisa não podem ser generalizados para o Brasil, uma vez que as condiçôes culturais, tecnológicas e financeiras dos atores envolvidos são pontuadas em diferentes níveis e graus de maturidade; b) os resultados devem ser observados em pessoas que foram consideradas respondentes, e c) é fundamental também destacar que o processo de adoção de tecnologia também pode ser observado em outras vertentes, o que pode limitar a generalização do fenômeno.

Evidenciam-se também sugestôes para futuras pesquisas: a) envolver questôes relativas à frequência de compra e uso de produtos/serviços; b) tentar compreender o comportamento dos indivíduos que não adotam tecnologia frequentemente; c) estudar questôes de envolvimento e utilização de produtos e serviços tecnológicos, d) estudar diferenciais na hora da aquisição do produto/serviço e e) aprofundar questôes sobre posse e uso de tecnologia em relação a técnicas de entrevistas por e-mail e face a face.

\section{Referências bibliográficas}

BAGOZZI, R.; GÜRCHAN-CANLI, Z.; PRIESTER, J.R. The social psychology of consumer behavior. Buckingham, UK: Open University Press, 2002.

CARO, A. Fatores críticos do comportamento do consumidor on line: um estudo exploratório. 2005. 166 f. Dissertação (Mestrado em Administração) - Faculdade de Economia e Administração e Contabilidade, Universidade de São Paulo, 2005.

CERVO, A.L.; BERVIAN, P.A. Metodologia científica. 5. ed. São Paulo: Prentice Hall, 2002.

CHRISTENSEN, C. The innovator's dilemma. New York: Harvard School Press, 1997. 
CORTADA, J.W. Making the information society: experience, consequences, and possibilities. Upper Saddle River: Prentice Hall, 2002.

DOSI, G. et al. Technical change and economic theory. London: Printer, 1988.

ENGEL, J. F. Comportamento do consumidor. São Paulo: LTC, 2000.

FREEMAN, C.; PEREZ, C. Structural crises of adjustment: business cycles and investment behaviour. In: DOSI, G. et al. (Ed.). Technical change and economic theory. London: Pinter, 1988. p. 38-66.

GRANT, R. M. Contemporary strategy analysis. 3rd ed. Malden: Blackwell, 1998.

HAIR JUNIOR, J. F. et al. Análise multivariada de dados. 5. ed. Porto Alegre: Bookman, 2005.

HAWKINS, D.I. et al. Consumer behavior: building marketing strategy. 8th ed. Boston: Irwin/McGraw Hill, 2001.

HOYER, W.; MACINNIS, D. Consumer behavior. 2nd ed. Boston: Houghton Mifflin, 2001.

IBGE. Cidades@. 2005. Disponível em: <http://www.ibge.com.br/cidadesat/default.php>. Acesso em: 26 jun. 2006.

JEUNON, E. E. Valores e orientação de consumo: proposta e validação de um modelo integrativo. In: ENCONTRO ANUAL DA ASSOCIAÇÃO NACIONAL DE PÓSGRADUAÇÃO EM ADMINISTRAÇÃO, 29., 2005, Brasília, DF. Anais... Brasília, DF: ANPAD, 2005. CD-ROM.

JOERGES, B. Technology in everyday life: conceptual queries. Journal of the Theory of Social Behavior, v. 2, n. 2, p. 219-237, 1988. Published on line.

JUDGE, P.C. Are tech buyers different? Business Week, 26 Jan. 1998. Disponível em: <http://www.businessweek.com/archives/1998/b3562090.arc.htm?campaign_id=search>. Acesso em: 10 fev. 2007.

KOTLER, P. Administração de marketing. São Paulo: Prentice Hall, 2000.

LEECH, N.L.; BARRET, K.C.; MORGAN, G.A. SPSS for Intermediate Statistics: Use and Interpretation. Second Edition. LEA: New Jersey, 2005.

SPSS for Introdutory Statistics: Use and Interpretation. Second Edition. LEA: New Jersey, 2004.

MALHOTRA, N.K. Pesquisa de marketing: uma orientação aplicada. São Paulo: Bookman, 2001. 
Luciana Peixoto Santa Rita, Alcides Carlos de Araújo, Mainah Almeida de Paula, Manuella de Oliveira Lima, José Carlos Viana Filho

MOORE, G. Crossing the chasm marketing and selling high-tech products to mainstream customers. New York: Harperbusiness, 1995.

MOWEN, I.C.; MINOR, M.S. Comportamento do consumidor. São Paulo: Prentice Hall, 2003.

NELSON, R.; WINTER, S. An evolutionary theory of economic change. Cambridge: Belknap, 1982.

PARASURAMAN, A. Technology Readiness Index (TRI): a multiple-item scale to measure readiness to embrace new technologies. Journal of Service Research, Thousand Oaks, v. 2, n. 4, p. 307-320, May 2000.

PARASURAMAN, A.; COLBY, C.L. Marketing para produtos inovadores: como e por que seus clientes adotam tecnologia. Porto Alegre: Bookman, 2002.

.. Techno-ready marketing: how and why your customers adopt technology. New York: Free Press, 2001.

PATEL, P.; PAVITT, K. The continuing, widespread (and neglected) importance of improvements in mechanical technologies. Research Policy, v. 23, p. 533-545, 1994

PAVITT, K. What we know about the Strategic Management of Technology. California Management Review, California, v. 32, n. 4, p. 17-26, Spring, 1990.

Sectoral Patterns of Technical Change: Toward a Taxonomy and a Theory. Research Policy, v. 13, n. 6, p. 343-373, Dec. 1984.

ROBERTSON, T.S. Em prol da revitalização. In: FINANCIAL times: dominando administração. São Paulo: Makron, 1999. p. 193-199.

Innovative behavior and communication. New York: Holt, Rinehart and Winston, 1971.

ROGERS, E.M. Diffusion of innovations. 5 ed. New York: Free Press, 2003.

Diffusion of innovations. 5. ed. New York: Free Press, 1995.

ROSENBERG, N. Perspectives on Technology. Cambridge UP: London, 1976.

ROTHWELL, R. Industrial innovation: success, strategy, trends. In: DODGSON, M.; ROTHWELL, R. The handbook of industrial innovation. Cheltenham: Edward Elgar, p. 33-53, 1995.

SCHIFFMAN, L.G.; KANUK, L.L. Comportamento do consumidor. 6. ed. Rio de Janeiro: LTC, 2000.

SOLOMON, M.R. Consumer behavior: buying, having and being. $4^{\text {th }}$ ed. New Jersey: Prentice Hall, 1998. 
SOUZA, R.V. Adoção de produtos e serviços baseados em tecnologia pelo consumidor: uma avaliação da aplicabilidade da technology readiness index no contexto brasileiro. 2002. 114 f. Dissertação (Mestrado em Administração) - Universidade Federal do Rio Grande do Sul, Porto Alegre, 2002.

TSIKRIKTSIS, N. A technology readiness-based taxonomy of customers: a replication and extension. Journal of Service Research, v. 7, n. 1, p. 42-52, 2004. Published on line. Disponível em: <http://jsr.sagepub.com/cgi/content/abstract/7/1/42>. Acesso em: 20 out. 2006.

TUSHMAN, M.; NADLER, D. Organizando-se para a inovação. In: STARKEY, K. Como as organizações aprendem: relatos do sucesso das grandes empresas. São Paulo: Futura, 1997. p. 166-189.

ENDEREÇOS PARA CORRESPONDÊNCIA:

Luciana Peixoto Santa Rita - Isantarita@hotmail.com

Alcides Carlos de Araújo - alcides.carlos@yahoo.com.br

Mainah Almeida de Paula - mainahalmeida@gmail.com

Manuella de Oliveira Lima - manuellalim@gmail.com

Rua Emp. Carlos da Silva Nogueira, 986, apto, 101

Ed. Villa Del Mare, Jatiuca,

Maceió-AL

57.036 .540

José Carlos Viana Filho - j_carlosadm@yahoo.com.br

Lot. Res. Dona Laura Vitalino, QD C, nº 09

Messias - AL

57990-000

08291289109 